\title{
異方性多層厚肉回転対称殼のクリープ変形*
}

\author{
竹園 茂 男*1, 右 田康 治*2
}

\section{The Creep of Anisotropic Multi-layered Moderately Thick Shells of Revolution}

\author{
Shigeo TAKEZONO and Kohji MIGITA
}

\begin{abstract}
The paper describes an analytical formulation and a numerical solution of the creep problems of anisotropic multi-layered moderately thick shells of revolution with application to a cylindrical shell. The analytical formulation is developed by extending the Reissner-Naghdi theory for elastic shells with consideration given to the effect of shear deformation. For the constitutive relation, Hooke's law for orthotropic materials is used in the elastic deformation, and equations based on the orthotropic creep theory derived from the orthotropic plastic theory by Hill are employed in the creep range. The creep strains are related to the stresses by McVetty's equation having the thermal effect multiplier by Arrhenius. The basic differential equations derived are numerically solved by a finite difference method. As a numerical example, the creep of a two-layerd, anisotropic cylindrical shell composed of mild steel and stainless steel subjected to uniform internal pressure is analyzed. Numerical computations have been carried out for four cases of the combinations of the directions of the anisotropic principal axis. It is found from the computations that the internal force distributions and the deformation are significantly varied depending on the combinations of the directions in layers.
\end{abstract}

Key Words: Structural Analysis, Anisotropic Multilayered Thick Shells, Creep Deformation, Shear Deformation, FDM

\section{1. 緒 言}

回転対称殼のクリープ変形問題に関しては, 従来か ら, 球殼, 円すい殼, 円筒殼などの特定の殻のクリー プ変形ばかりでなく，一般の任意形状の軸対称殻の解 法を述べたものも多数報告されている(1) (7). また薄肉 の壳ばかりでなく, 比較的厚い殼についても二, 三の 研究がなされている(8)(9). しかしながら、これらの研究 は，単一材質加なるものを対象としており，近年広 く実用されている異種材質からなる殻のクリープに関 する研究は，あまり行われていないようである.

そこで, 著者らはこの研究に着手し,すでに薄肉の 多層回転殻や中程度の厚さの多層軸対称殼に一般の非 対称な荷重が作用する場合について解析を行ってきた が(10)(11), 本研究では, 異方性主軸の方向の異なる層を 重ねた多層回転対称殼のクリープ変形問題を取扱っ た.クリープの構成関係には, 温度効果をアレニウス の式で表した McVetty の式(12)を, Hill の直交異方性 塑性理論にならって多軸応力状態に拡張したものを用 いた，厚肉の場合に考慮すべきせん断変形には，Reis-

\footnotetext{
* 昭和 63 年 7 月 15 日 東海支部浜松地方講演会において講 演, 原稿受付 昭和 62 年 10 月 22 日.

*1 正員, 豊橋技術科学大学 (《 440 豊橋市天伯町).

*2 准員, 三菱重工 (株) 長㱦造船所 (⿶850 長崎市飽ノ浦町).
}

sner-Naghdi の理論(13)(14)を適用して基礎式を導き， これを差分法によって数值解析した。

数值例として, 実用上重要な二層構造の円筒殸に, 一様内圧が作用する問題を取り上げ, 変形および内力 の時間的変化の樣子を解析した。

\section{2. 解 法}

$2 \cdot 1$ 基礎式 図1に示すように, 対称軸から殼 の中央面上の任意点 $\mathrm{P}$ までの距離を $r$ とし, 款の中 央面の形を $r=r(s)$ で与えると, 中央面の点 $\mathrm{P} に お$ ける無次元曲率 $\omega_{\xi}\left(=a / R_{s}\right), \omega_{\theta}\left(=a / R_{\theta}\right)$ および無次元 半径 $\rho(=r / a)$ の間には次の関係が成立する.ただし, $s$ は殼の子午線方向の長さであり, $a$ は代表長さであ る.

$$
\begin{aligned}
& \omega_{\xi}=-\left(\gamma^{\prime}+\gamma^{2}\right) / \omega_{\theta}, \omega_{\theta}=\sqrt{1-\left(\rho^{\prime}\right)^{2}} / \rho \\
& \omega_{\theta}^{\prime}=\gamma\left(\omega_{\xi}-\omega_{\theta}\right), \rho^{\prime \prime} / \rho=-\omega_{\xi} \omega_{\theta} \\
& \gamma=\rho^{\prime} / \rho, \xi=s / a,()^{\prime}=d(\quad) / d \xi
\end{aligned}
$$

また, 殸の任意点を表すのに, 直交座標 $(\xi, \theta, \zeta)$ を用

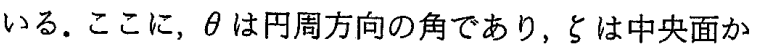
ら外向きに測った長さである。

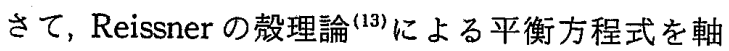
対称殼に適用し，時間または時間とともに単調に変化 
する量について微分すると，次のようになる.

$$
\begin{aligned}
& \frac{\partial \dot{N}_{\xi}}{\partial \xi}+\gamma\left(\dot{N}_{\xi}-\dot{N}_{\theta}\right)+\frac{1}{\rho} \frac{\partial \dot{N}_{\theta \xi}}{\partial \theta}+\omega_{\xi} \dot{Q}_{\xi}+a \dot{P}_{\xi}=0 \\
& \frac{\partial \dot{N}_{\xi \theta}}{\partial \xi}+\gamma\left(\dot{N}_{\xi \theta}+\dot{N}_{\theta \xi}\right)+\frac{1}{\rho} \frac{\partial \dot{N}_{\theta}}{\partial \theta}+\omega_{\theta} \dot{Q}_{\theta}+a \dot{P}_{\theta}=0 \\
& \frac{\partial \dot{Q}_{\xi}}{\partial \xi}+\gamma \dot{Q}_{\xi}+\frac{1}{\rho} \frac{\partial \dot{Q}_{\theta}}{\partial \theta}-\left(\omega_{\xi} \dot{N}_{\xi}+\omega_{\theta} \dot{N}_{\theta}\right)+a \dot{P}_{\xi}=0 \\
& \dot{Q}_{\xi}-\frac{1}{a}\left[\frac{\partial \dot{M}_{\xi}}{\partial \xi}+\gamma\left(\dot{M}_{\xi}-\dot{M}_{\theta}\right)+\frac{1}{\rho} \frac{\partial \dot{M}_{\theta \xi}}{\partial \theta}\right]=0 \\
& \dot{Q}_{\theta}-\frac{1}{a}\left[\frac{\partial \dot{M}_{\xi \theta}}{\partial \xi}+\gamma\left(\dot{M}_{\xi \theta}+\dot{M}_{\theta \xi}\right)+\frac{1}{\rho} \frac{\partial \dot{M}_{\theta}}{\partial \theta}\right]=0
\end{aligned}
$$

ここに, $N_{\xi}, N_{\theta}, N_{\xi \theta}, N_{\theta \xi}, Q_{\xi}, Q_{\theta}$ おるよび $M_{\xi}, M_{\theta}, M_{\xi \theta}$, $M_{0 \xi}$ は図 1 に示すように壳の単位長さ当たりの合応力 および合モーメントである. $P_{\xi}, P_{\theta}, P_{5}$ は殻の中央面の 单位面積当たりの分布荷重であり，殼の内外面に作用 する分布荷重 $\left\{P_{\xi}^{-}, P_{\theta}^{-}, P_{\xi}^{-}\right\},\left\{P_{\xi}^{+}, P_{\theta}^{+}, P_{\xi}^{+}\right\}$と次の関係 にある。

$$
\left\{P_{\xi}, P_{\theta}, P_{\xi}\right\}=\left\{P_{\xi}^{-}, P_{\theta}^{-}, P_{\xi}^{-}\right\} h^{-}-\left\{P_{\xi}^{+}, P_{\theta}^{+}, P_{\xi}^{+}\right\} h^{+}
$$

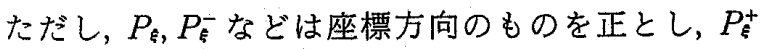
などはこれと反対方向のものを正とする，また，

$$
h^{ \pm}=1 \pm \frac{h}{2}\left(\frac{1}{R_{s}}+\frac{1}{R_{\theta}}\right)+\frac{h^{2}}{4} \frac{1}{R_{s} R_{\theta}}
$$

次に中央面のひずみ $\varepsilon_{\xi m,} \varepsilon_{\theta m}$ および $\varepsilon_{\xi \theta m}$ を $\xi, \theta, \zeta$ 方向の変位 $U_{\xi}, U_{\theta}, U_{\xi}$ で表すと,

$$
\left.\begin{array}{l}
\dot{\varepsilon}_{\xi m}=\frac{1}{a}\left[\frac{\partial \dot{U}_{\xi}}{\partial \xi}+\omega_{\xi} \dot{U}_{\zeta}\right] \\
\dot{\varepsilon}_{\theta m}=\frac{1}{a}\left[\frac{1}{\rho} \frac{\partial \dot{U}_{\theta}}{\partial \theta}+\gamma \dot{U}_{\xi}+\omega_{\theta} \dot{U}_{\xi}\right] \\
\dot{\varepsilon}_{\xi \theta m}=\frac{1}{2 a}\left[\frac{1}{\rho} \frac{\partial \dot{U}_{\xi}}{\partial \theta}+\frac{\partial \dot{U}_{\theta}}{\partial \xi}-\gamma \dot{U}_{\theta}\right]
\end{array}\right\}
$$

ここに, $\varepsilon_{\xi \theta m}$ は工学上のせん断ひずみの $1 / 2$ である.

また，曲率の変化量 $\chi_{\xi}, x_{\theta}, \chi_{\xi \theta}, x_{\theta \xi}$ と変位成分との 関係は次のようになる.

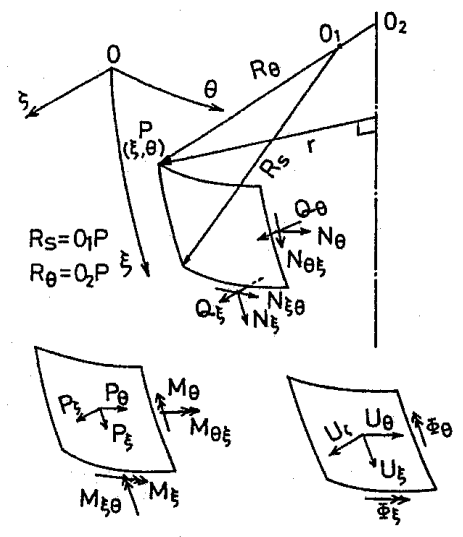

図 1 諸記号

$$
\left.\begin{array}{l}
\dot{x}_{\xi}=\frac{1}{a} \frac{\partial \dot{\Phi}_{\xi}}{\partial \xi}, \dot{x}_{\theta}=\frac{1}{a}\left(\frac{1}{\rho} \frac{\partial \dot{\Phi}_{\theta}}{\partial \theta}+\gamma \dot{\Phi}_{\xi}\right) \\
\dot{x}_{\xi \theta}=\frac{1}{2 a}\left(\frac{\partial \dot{\Phi}_{\theta}}{\partial \xi}-2 \omega_{\xi} \dot{\Phi}_{n}\right) \\
\dot{x}_{\theta \xi}=\frac{1}{2 a}\left(\frac{1}{\rho} \frac{\partial \dot{\Phi}_{\xi}}{\partial \theta}-\gamma \dot{\Phi}_{\theta}+2 \omega_{\theta} \dot{\Phi}_{n}\right)
\end{array}\right\}
$$

ここに, $\Phi_{\xi}, \Phi_{\theta}, \Phi_{n}$ は中央面の回転を示し，

$$
\left.\begin{array}{l}
\dot{\Phi}_{\xi}=\frac{1}{a}\left(-\frac{\partial \dot{U}_{\xi}}{\partial \xi}+\omega_{\xi} \dot{U}_{\xi}\right)+2 \dot{\varepsilon}_{\xi \zeta m} \\
\dot{\Phi}_{\theta}=\frac{1}{a}\left(-\frac{1}{\rho} \frac{\partial \dot{U}_{\zeta}}{\partial \theta}+\omega_{\theta} \dot{U}_{\theta}\right)+2 \dot{\varepsilon}_{\theta \zeta m} \\
\dot{\Phi}_{n}=\frac{1}{2 a}\left(-\frac{1}{\rho} \frac{\partial \dot{U}_{\xi}}{\partial \theta}+\frac{\partial \dot{U}_{\theta}}{\partial \xi}+\gamma \dot{U}_{\theta}\right)
\end{array}\right\}
$$

壳厚中央面から $\zeta$ 距離にある点 $\mathrm{Q}$ のひずみ速度 成分は次式で表される。

$$
\begin{aligned}
& \dot{\varepsilon}_{\xi}=\left(\dot{\varepsilon}_{\xi m}+\zeta \dot{x}_{\xi}\right) / L_{\xi}, \dot{\varepsilon}_{\theta}=\left(\dot{\varepsilon}_{\theta m}+\zeta \dot{x}_{\theta}\right) / L_{\theta} \\
& \dot{\varepsilon}_{\xi \theta}=\left\{\frac{1}{2}\left(\dot{\varepsilon}_{\xi \theta m}+\dot{\Phi}_{n}\right)+\zeta\left(\dot{x}_{\xi \theta}+\dot{\Phi}_{n} / R_{s}\right)\right\} / L_{\xi} \\
& +\left\{\frac{1}{2}\left(\dot{\varepsilon}_{\xi \theta m}-\dot{\Phi}_{n}\right)+\zeta\left(\dot{x}_{\theta \xi}-\dot{\Phi}_{n} / R_{\theta}\right)\right\} / L_{\theta} \\
& \dot{\varepsilon}_{\xi \zeta}=\dot{\varepsilon}_{\xi 5 m} / L_{\xi}, \dot{\varepsilon}_{\theta \zeta}=\dot{\varepsilon}_{\theta \zeta m} / L_{\theta}
\end{aligned}
$$

ただし， $L_{\xi}=1+\zeta / R_{s}, L_{\theta}=1+\zeta / R_{\theta}$

本研究では, 殼の各層の材料は直交異方性を有し, 異方性主軸 $(1,2)$ の方向は, 一般に壳の座標軸 $(\xi, \theta)$ 方向と異なるものとする（図 2 ）.

今, 各層の全ひずみ速度が，弾性ひずみ速度とクり 一プひずみ速度との和から成るものとすると, 全ひず み速度は, 次式で表される.ただし, 殸厚方向の垂直応 力は省略する。

$$
\{\dot{\varepsilon}\}=[D]^{-1}\{\dot{\sigma}\}+\left\{\dot{\varepsilon}^{c}\right\}+\left\{\dot{\varepsilon}^{t}\right\}
$$
ここに,

$$
\begin{aligned}
& \{\dot{\varepsilon}\}=\left\{\dot{\varepsilon}_{1}, \dot{\varepsilon}_{2,}, \dot{\varepsilon}_{12}, \dot{\varepsilon}_{13}, \dot{\varepsilon}_{23}\right\}^{T} \\
& \{\dot{\sigma}\}=\left\{\dot{\sigma}_{1}, \dot{\sigma}_{2}, \dot{\sigma}_{12}, \dot{\sigma}_{13}, \dot{\sigma}_{23}\right\}^{T} \\
& \left\{\dot{\varepsilon}^{c}\right\}=\left\{\dot{\varepsilon}_{1}^{c}, \dot{\varepsilon}_{2}^{c}, \dot{\varepsilon}_{12,}^{c} \dot{\varepsilon}_{13}^{c}, \dot{\varepsilon}_{23}^{c}\right\}^{T} \\
& \left\{\dot{\varepsilon}^{t}\right\}=\left\{\alpha_{1} \dot{T}_{e}, \alpha_{2} \dot{T}_{e}, 0,0,0\right\}^{T} \\
& {[D]^{-1}=\left[\begin{array}{cccc}
1 / E_{1}-\nu_{12} / E_{1} & 0 & 0 & \\
1 / E_{2} & 0 & 0 & 0 \\
& 1 / 2 G_{12} & 0 & 0 \\
& & 1 / 2 G_{13} & 0 \\
\text { Sym. } & & & 1 / 2 G_{23}
\end{array}\right]}
\end{aligned}
$$

上式で， $E_{1}, E_{2}$ は縦弾性係数, $G_{12}, G_{13}, G_{23}$ はせん断 弾性係数, $\nu_{12}$ はポアソン比, $\alpha_{1}, \alpha_{2}$ は線膨張係数であ る.また $T_{e}$ は基準温度 $T_{0}$ からの温度変化で, 殸の任 意点の温度を $T$ とすると次式となる。

$T_{e}(\xi, \theta, \zeta, t)=T(\xi, \theta, \zeta, t)-T_{0}$

さて, 一定の単軸応力下におけるクリープひずみが, 
応力と時間に関しては McVetty の式に，また温度依 存性についてはアレニウスの式によるものとすると,

$$
\varepsilon^{c}=A e^{-\beta / T} \sigma^{m}\left(1-e^{-q t}+c t\right)
$$

ここに, $A, m, q, c, \beta$ は材料の特性值, $T$ は絶対温度 である。

上式を, Hill の直交異方性理論を用いて, 直交異方 性の場合に拡張すると，時間硬化則に基づく各ひずみ 速度成分は次のようになる，ただし，殼厚方向の垂直 応力は省略する。

$$
\left\{\dot{\varepsilon}^{c}\right\}=A e^{-\beta / T} \bar{\sigma}^{m-1}\left(q e^{-q t}+c\right)[B](\dot{\sigma}\}
$$

ここに,

$$
[B]=\frac{3}{2} \frac{1}{F+G+H}\left[\begin{array}{ccccc}
G+H & -H & 0 & 0 & 0 \\
-H & F+H & 0 & 0 & 0 \\
0 & 0 & N & 0 & 0 \\
0 & 0 & 0 & M & 0 \\
0 & 0 & 0 & 0 & L
\end{array}\right]
$$

̄ は相当応力で, 次式で表される.

$$
\begin{aligned}
\bar{\sigma} & =\sqrt{\frac{3}{2(F+G+H)}}\left[(G+H) \sigma_{1}^{2}-2 H \sigma_{1} \sigma_{2}\right. \\
& \left.+(F+H) \sigma_{2}^{2}+2 L \sigma_{23}^{2}+2 M \sigma_{13}^{2}+2 N \sigma_{12}^{2}\right]^{1 / 2}
\end{aligned}
$$

式(14)，(15)において, $F, G, H, L, M, N$ 注材料の異 方性パラメータであり, 等方性の場合には, $F=G=$ $H=1, L=M=N=3$ となる.

次に，式 $(9)$ を款の座標 $(\xi, \theta, \zeta)$ に変換する。変換 マトリックスを $[M]$ とすると，

$$
\{\dot{\bar{\varepsilon}}\}=[\bar{D}]^{-1}\{\dot{\bar{\sigma}}\}+\left\{\dot{\bar{\varepsilon}}^{c}\right\}+\left\{\dot{\bar{\varepsilon}}^{t}\right\}
$$
ここに,

$$
\begin{aligned}
& \{\dot{\bar{\varepsilon}}\}=[M]\{\dot{\varepsilon}\} \\
& \left\{\dot{\bar{\varepsilon}}^{c}\right\}=[M]\left\{\dot{\varepsilon}^{c}\right\} \\
& \left\{\dot{\bar{\varepsilon}}^{t}\right\}=[M]\left\{\dot{\varepsilon}^{t}\right\} \\
& \{\dot{\bar{\sigma}}\}=[M]\{\dot{\sigma}\} \\
& {[\bar{D}]^{-1}=[M][D]^{-1}[M]^{-1}}
\end{aligned}
$$

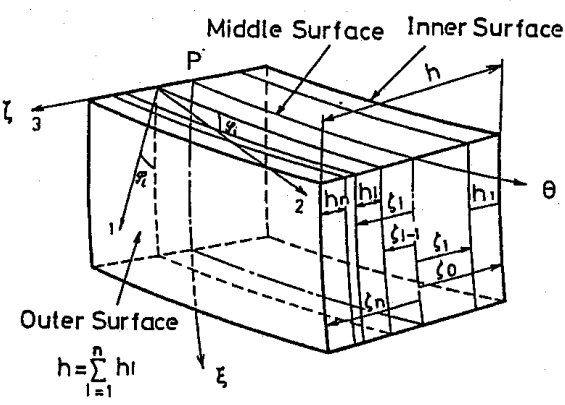

図 2 異方性多層殼要素

$$
\left.\begin{array}{l}
{[M]=\left[\begin{array}{ccccc}
\alpha^{2} & \beta^{2} & 2 \alpha \beta & 0 & 0 \\
\beta^{2} & \alpha^{2} & -2 \alpha \beta & 0 & 0 \\
-\alpha \beta & \alpha \beta & \alpha^{2}-\beta^{2} & 0 & 0 \\
0 & 0 & 0 & \alpha & \beta \\
0 & 0 & 0 & -\beta & \alpha
\end{array}\right]} \\
\alpha=\cos \varphi, \beta=\sin \varphi \\
\{\dot{\bar{\varepsilon}}\}=\left\{\dot{\varepsilon}_{\xi}, \dot{\varepsilon}_{\theta}, \dot{\varepsilon}_{\xi \theta,}, \dot{\varepsilon}_{\xi \zeta}, \dot{\varepsilon}_{\theta \zeta}\right\}^{T} \\
\left\{\dot{\bar{\varepsilon}}^{c}\right\}=\left\{\dot{\varepsilon}_{\xi}^{c}, \dot{\varepsilon}_{\theta,}^{c} \dot{\varepsilon}_{\xi \theta}^{c}, \dot{\varepsilon}_{\xi \zeta}^{c}, \dot{\varepsilon}_{\theta \zeta}^{c}\right\}^{T} \\
\left\{\dot{\bar{\varepsilon}}^{t}\right\}=\left\{\dot{\varepsilon}_{\xi}^{t}, \dot{\varepsilon}_{\theta}^{t}, \dot{\varepsilon}_{\xi \theta}^{t}, 0,0\right\}^{T} \\
\{\dot{\bar{\sigma}}\}=\left\{\dot{\sigma}_{\xi}, \dot{\sigma}_{\theta}, \dot{\sigma}_{\xi \theta}, \dot{\sigma}_{\xi \zeta}, \dot{\sigma}_{\theta \zeta}\right\}^{T}
\end{array}\right\}
$$

式(16)を応力について解くと,

$$
\{\dot{\bar{\sigma}}\}=[\bar{D}]\{\dot{\bar{\varepsilon}}\}-\left\{\dot{\bar{\sigma}}^{c}\right\}-\left\{\dot{\bar{\sigma}}^{t}\right\}
$$

ここに,

$$
\left\{\dot{\bar{\sigma}}^{c}\right\}=[\bar{D}]\left\{\dot{\bar{\varepsilon}}^{c}\right\},\left\{\dot{\bar{\sigma}}^{t}\right\}=[\bar{D}]\left\{\dot{\bar{\varepsilon}}^{t}\right\}
$$

壳の単位長さ当たりの合応力および合モーメント は，式(18)を用いると，次のようになる（図 2)。なお， 添字 $i$ は殻の $i$ 層を意味する.

$$
\begin{aligned}
& \left\{\begin{array}{l}
\dot{N} \\
\dot{M}
\end{array}\right\}=\int_{-h / 2}^{h / 2}[L]\left\{\begin{array}{c}
\dot{\bar{\sigma}} \\
\dot{\bar{\sigma}} * \zeta
\end{array}\right\} d \zeta \\
& \quad=\sum_{i=1}^{n} \int_{\zeta_{i-1}}^{\zeta_{i}}[L]\left[\begin{array}{cc}
\bar{D}_{i} & 0 \\
0 & \bar{D}_{i}^{\prime}
\end{array}\right]\left\{\begin{array}{c}
\dot{\bar{\varepsilon}} \\
\dot{\bar{\varepsilon}}^{*} \zeta
\end{array}\right\} d \zeta-\left\{\begin{array}{l}
\dot{N}^{c} \\
\dot{M}^{c}
\end{array}\right\}-\left\{\begin{array}{l}
\dot{N}^{t} \\
\dot{M}^{t}
\end{array}\right\}
\end{aligned}
$$

ここで,

$$
\begin{aligned}
& \{\dot{N}\}=\left\{\dot{N}_{\xi}, \dot{N}_{\theta}, \dot{N}_{\xi \theta}, \dot{N}_{\theta \xi}, \dot{Q}_{\xi}, \dot{Q}_{\theta}\right\}^{T} \\
& \{\dot{M}\}=\left\{\dot{M}_{\xi}, \dot{M}_{\theta}, \dot{M}_{\xi \theta}, \dot{M}_{\theta \xi}\right\}^{T}
\end{aligned}
$$

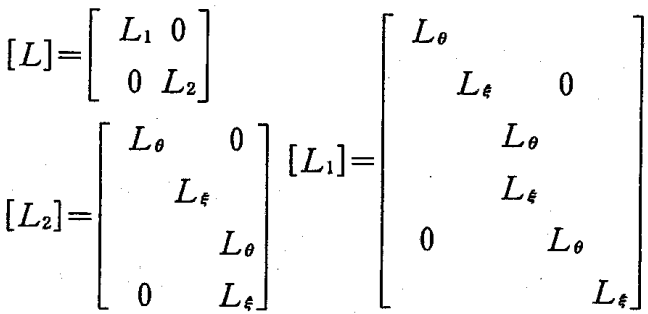$$
\left(\left\{a_{1}, a_{2}, a_{3}, a_{4}, a_{5}\right\}^{T}\right)^{*}=\left\{a_{1}, a_{2}, a_{3}\right\}^{T}
$$

また，

$\left\{\dot{N}^{c}\right\}=\left\{\dot{N}_{\xi}^{c}, \dot{N}_{\theta}^{c}, \dot{N}_{\xi \theta}^{c}, \dot{N}_{\theta \xi}^{c}, \dot{Q}_{\xi}^{c}, \dot{Q}_{\theta}^{c}\right\}^{T}$

$=\int_{-h / 2}^{h / 2}\left[L_{1}\right]\left\{\dot{\bar{\sigma}}^{c}\right\} d \zeta=\sum_{i=1}^{n} \int_{\zeta_{i-1}}^{\zeta_{i}}\left[L_{1}\right]\left[\bar{D}_{i}\right]\left\{\dot{\bar{\varepsilon}}_{i}^{c}\right\} d \zeta$

$\left\{\dot{N}^{t}\right\}=\left\{\dot{N}_{\xi}^{t}, \dot{N}_{\theta}^{t}, \dot{N}_{\xi \theta}^{t}, \dot{N}_{\theta \xi}^{t}, 0,0\right\}^{T}$

$=\int_{-h / 2}^{h / 2}\left[L_{1}\right]\left\{\dot{\bar{\sigma}}^{t}\right\} d \zeta=\sum_{i=1}^{n} \int_{\zeta_{i-1}}^{\zeta_{i}}\left[L_{1}\right]\left[\bar{D}_{i}\right]\left\{\dot{\bar{\varepsilon}}_{i}^{t}\right\} d \zeta$

$\left\{\dot{M}^{c}\right\}=\left\{\dot{M}_{\xi}^{c}, \dot{M}_{\theta}^{c}, \dot{M}_{\xi \theta}^{c}, \dot{M}_{\theta \xi}^{c}\right\}^{T}$

$=\int_{-h / 2}^{h / 2}\left[L_{2}\right]\left\{\dot{\sigma}^{c}\right\} * \zeta d \zeta$

$=\sum_{i=1}^{n} \int_{\zeta_{i-1}}^{\zeta_{i}}\left[L_{2}\right]\left[\bar{D}_{i}^{\prime}\right]\left\{\dot{\varepsilon}_{i}^{c}\right]^{*} \zeta d \zeta$

$\left\{\dot{M}^{t}\right\}=\left\{\dot{M}_{\xi}^{t}, \dot{M}_{\theta}^{t}, \dot{M}_{\xi \theta}^{t}, \dot{M}_{\theta \xi}^{t}\right\}^{T}$ 
$=\int_{-h / 2}^{h / 2}\left[L_{2}\right]\left\{\dot{\sigma}^{t}\right\} * \zeta d \zeta$

$=\sum_{i=1}^{n} \int_{\xi_{t-1}}^{t_{t}}\left[L_{2}\right]\left[\bar{D}_{i}^{\prime}\right]\left\{\dot{\bar{\varepsilon}}_{i}^{t}\right\}^{*} \zeta d \zeta$

ここに,

$$
\left[\bar{D}_{i}\right]=\left[\begin{array}{ccc}
\bar{D}_{i}^{\prime} & 0 & 0 \\
0 & \bar{d}_{44 i} & \bar{d}_{45 i} \\
0 & \bar{d}_{54 i} & \bar{d}_{55 i}
\end{array}\right],\left[\bar{D}_{i}^{\prime}\right]=\left[\begin{array}{ccc}
\bar{d}_{11 i} & \bar{d}_{12 i} & \bar{d}_{13 i} \\
\bar{d}_{21 i} & \bar{d}_{22 i} & \bar{d}_{23 i} \\
\bar{d}_{31 i} & \bar{d}_{32 i} & \bar{d}_{33 i}
\end{array}\right]
$$

要素 $\bar{d}_{11 i}$ などは殼の各層の弾性定数, 各層の異方性 主軸 $(1,2)$ と壳の座標軸 $(\xi, \theta)$ とのなす角 $\varphi_{i}$ から求

\section{められる。}

式(20)に式 (8)を代入し，積分の際，近似，

$$
\frac{L_{\theta}}{L_{\xi}} \simeq 1-\left(\frac{1}{R_{s}}-\frac{1}{R_{\theta}}\right) \zeta+\frac{1}{R_{s}}\left(\frac{1}{R_{s}}-\frac{1}{R_{\theta}}\right) \zeta^{2}
$$

を用いると, 式(20)は

$$
\left\{\begin{array}{l}
\dot{N} \\
\dot{M}
\end{array}\right\}=[A]\left\{\begin{array}{c}
\dot{\varepsilon}_{m} \\
\dot{x} \\
\dot{\Phi}_{n}
\end{array}\right\}-\left\{\begin{array}{l}
\dot{N}^{c} \\
\dot{M}^{c}
\end{array}\right\}-\left\{\begin{array}{l}
\dot{N}^{t} \\
\dot{M}^{t}
\end{array}\right\}
$$

ここに,

$$
\left.\begin{array}{l}
\left\{\dot{\varepsilon}_{m}\right\}=\left\{\dot{\varepsilon}_{\xi m}, \dot{\varepsilon}_{\theta m .}, \dot{\varepsilon}_{\xi \theta m}, \dot{\varepsilon}_{\varepsilon \zeta m}, \dot{\varepsilon}_{\theta 5 m}\right\} \\
\{\dot{x}\}=\left\{\dot{x}_{\xi}, \dot{x}_{\theta}, \dot{x}_{\xi \theta}, \dot{x}_{\theta \xi}\right\}
\end{array}\right\}
$$

$$
\left.\begin{array}{l}
(a)=\frac{1}{2}\left(\zeta_{1, i}^{*}-\zeta_{1, i}\right)+\frac{\omega_{\xi}}{a} \zeta_{2, i}^{*}-\frac{\omega_{\theta}}{a} \zeta_{2, i},(b)=\frac{1}{2}\left(\zeta_{1, i}-\zeta_{1, i}^{* *}\right)+\frac{\omega_{\xi}}{a} \zeta_{2, i}-\frac{\omega_{\theta}}{a} \zeta_{2, i}^{* *} \\
(c)=\frac{1}{2}\left(\zeta_{2, i}^{*}-\zeta_{2, i}\right)+\frac{\omega_{\xi}}{a} \zeta_{3, i}^{*}-\frac{\omega_{\theta}}{a} \zeta_{3, i},(d)=\frac{1}{2}\left(\zeta_{2, i}-\zeta_{2, i}^{* *}\right)+\frac{\omega_{\xi}}{a} \zeta_{3, i}-\frac{\omega_{\theta}}{a} \zeta_{3, i}^{* *} \\
\zeta_{n, i}=\frac{1}{n}\left(\zeta_{i}^{n}-\zeta_{i-1}^{n}\right)(n=1,2, \cdots, 5) \\
\zeta_{n, i}^{*}=\zeta_{n, i}-\frac{1}{a}\left(\omega_{\xi}-\omega_{\theta}\right) \tau_{n+1, i}+\frac{\omega_{\xi}}{a^{2}}\left(\omega_{\xi}-\omega_{\theta}\right) \zeta_{n+2, i}, \zeta_{n, i}^{* *}=\zeta_{n, i}-\frac{1}{a}\left(\omega_{\theta}-\omega_{\xi}\right) \zeta_{n+1, i}+\frac{\omega_{\theta}}{a^{2}}\left(\omega_{\theta}-\omega_{\xi}\right) \zeta_{n+2, i} \\
\quad(n=1,2,3)
\end{array}\right\}
$$

以上で 64 個の独立変数 $\dot{U}_{\xi}, \dot{U}_{\theta}, \dot{U}_{\xi}, \dot{\Phi}_{\varepsilon}, \dot{\Phi}_{\theta}, \dot{\Phi}_{n},\{\dot{N}\},\left\{\dot{N}^{c}\right\},\left\{\dot{N}^{T}\right\},\{\dot{M}\},\left\{\dot{M}^{c}\right\},\left\{\dot{M}^{T}\right\},\left\{\dot{\varepsilon}_{m}\right\},\{\dot{x}\},\left\{\dot{\bar{\varepsilon}}^{c}\right\},\left\{\dot{\bar{\varepsilon}}^{t}\right\},\{\dot{\bar{\sigma}}\}$, $\left\{\dot{\sigma}^{c}\right\},\left\{\dot{\sigma}^{t}\right\}$ に対し，式(2)，(5)〜 (7)，(16)，(17)，(19)，(21)，(23)の64 個の式が得られたことになる.なお上 式で, $n=1$ とおくと, 単層殻の場合に, $\varphi_{i}=0$ とおくと, 直交異方性の殸の場合に, また 1,2 方向の弾性係数と線 膨張係数を等しく置き, クリープの異方性パラメータを $F=G=H=1, L=M=N=3$ とすると, 等方性の場合に 㷌着する。

$2 \cdot 2$ 無次元方程式 一般に非対称問題を解析するには, 各定数を $\theta$ 方向にフーリエ級数で表し, その係数を 定めればよい.そこで上に示した 64 個の変数のうち, $\left\{\dot{\bar{\varepsilon}}^{c}\right\},\left\{\dot{\bar{\varepsilon}}^{t}\right\}$ を除く 56 個の変数を次のようにフーリエ級数で表 す.

$\left\{\dot{N}_{\xi}, \dot{N}_{\xi}^{c}, \dot{N}_{\xi}^{t}, \dot{N}_{\theta}, \dot{N}_{\theta}^{c}, \dot{N}_{\theta}^{t}, \dot{N}_{\xi \theta}, \dot{N}_{\xi}^{c}, \dot{N}_{\xi \theta}^{t}, \dot{N}_{\theta \xi}, \dot{N}_{\theta \xi}^{c}, \dot{N}_{\theta \xi}^{t}, \dot{Q}_{\xi}, \dot{Q}_{\xi}^{c}, \dot{Q}_{\theta}, \dot{Q}_{\theta}^{c}\right\}$

$=\sigma_{0} h_{0} \sum_{n=0}^{\infty}\left\{\dot{n}_{\xi}^{(n)}, \dot{n}_{\xi}^{c(n)}, \dot{n}_{\xi}^{t(n)}, \dot{n}_{\theta}^{(n)}, \dot{n}_{\theta}^{c(n)}, \dot{n}_{\theta}^{t(n)}, \dot{\bar{n}}_{\xi \theta}^{(n)}, \dot{\bar{n}}_{\xi \theta}^{c(n)}, \dot{\bar{n}}_{\xi \theta}^{t(n)}, \dot{\bar{n}}_{\theta \xi}^{(n)}, \dot{\bar{n}}_{\theta \xi}^{c(n)}, \dot{\bar{n}}_{\theta \xi}^{t(n)}, \dot{q}_{\xi}^{(n)}, \dot{q}_{\xi}^{(n)}, \dot{\bar{q}}_{\theta}^{(n)}, \dot{\bar{q}}_{\theta}^{c(n)}\right\}$

$\times \cos n \theta$

$+\sigma_{0} h_{0} \sum_{n=1}^{\infty}\left\{\dot{\bar{n}}_{\xi}^{(n)}, \dot{\bar{n}}_{\xi}^{c(n)}, \dot{\bar{n}}_{\xi}^{t(n)}, \dot{\bar{n}}_{\theta}^{(n)}, \dot{\bar{n}}_{\theta}^{c(n)}, \dot{\bar{n}}_{\theta}^{t(n)}, \dot{n}_{\xi \theta}^{(n)}, \dot{n}_{\xi \theta}^{c(n)}, \dot{n}_{\xi \theta}^{t(n)}, \dot{n}_{\theta \xi}^{(n)}, \dot{n}_{\theta \xi}^{c(n)}, \dot{n}_{\theta \xi}^{t(n)}, \dot{\bar{q}}_{\xi}^{(n)}, \dot{\bar{q}}_{\xi}^{(n)}, \dot{q}_{\theta}^{(n)}, \dot{q}_{\theta}^{c(n)}\right\}$

$\times \sin n \theta$

$\left\{\dot{M}_{\xi}, \dot{M}_{\xi}^{c}, \dot{M}_{\xi}^{t}, \dot{M}_{\theta}, \dot{M}_{\theta}^{c}, \dot{M}_{\theta}^{t}, \dot{M}_{\xi \theta}, \dot{M}_{\xi \theta}^{c}, \dot{M}_{\xi \theta}^{t}, \dot{M}_{\theta \xi}, \dot{M}_{\theta \xi}^{c}, \dot{M}_{\theta \xi}^{t}\right\}$ 
$=\left(\sigma_{0} h_{0}^{3} / a\right) \sum_{n=0}^{\infty}\left\{\dot{m}_{\xi}^{(n)}, \dot{m}_{\xi}^{c(n)}, \dot{m}_{\varepsilon}^{t(n)}, \dot{m}_{\theta}^{(n)}, \dot{m}_{\theta}^{c(n)}, \dot{m}_{\theta}^{t(n)}, \dot{\bar{m}}_{\xi \theta}^{(n)}, \dot{\bar{m}}_{\theta \theta}^{c(n)}, \dot{\bar{m}}_{\xi \theta}^{t}(n), \dot{\bar{m}}_{\theta \xi}^{(n)}, \dot{\bar{m}}_{\theta \varepsilon}^{c(n)}, \dot{\bar{m}}_{\theta \xi}^{t(n)}\right\} \cos n \theta$ $+\left(\sigma_{0} h_{0}^{3} / a\right) \sum_{n=1}^{\infty}\left\{\dot{\bar{m}}_{\xi}^{(n)}, \dot{\bar{m}}_{\xi}^{c(n)}, \dot{\bar{m}}_{\xi}^{t(n)}, \dot{\bar{m}}_{\theta}^{(n)}, \dot{\bar{m}}_{\theta}^{c(n)}, \dot{\bar{m}}_{\theta}^{t(n)}, \dot{m}_{\xi \theta}^{(n)}, \dot{m}_{\xi \theta}^{c(n)}, \dot{m}_{\xi \theta}^{t(n)}, \dot{m}_{\theta \xi}^{(n)}, \dot{m}_{\theta \xi}^{c(n)}, \dot{m}_{\theta \xi}^{t(n)}\right\} \sin n \theta$ $\left\{\dot{x}_{\xi}, \dot{x}_{\theta}, \dot{x}_{\xi \theta}, \dot{x}_{\theta \xi}\right\}=\left(\sigma_{0} / a E_{0}\right) \sum_{n=0}^{\infty}\left\{\dot{k}_{\xi}^{(n)}, \dot{k}_{\theta}^{(n)}, \dot{k}_{\xi \theta}^{(n)}, \dot{k}_{\theta_{\xi}(n)}^{(n)}\right\} \cos n \theta+\left(\sigma_{0} / a E_{0}\right) \sum_{n=1}^{\infty}\left\{\dot{k}_{\xi}^{(n)}, \dot{k}_{\theta}^{(n)}, \dot{k}_{\xi \theta}^{(n)}, \dot{k}_{\theta \xi}^{(n)}\right\} \sin n \theta$ $\left\{\dot{U}_{\xi}, \dot{U}_{\theta}, \dot{U}_{\xi}\right\}=\left(\sigma_{0} a / E_{0}\right) \sum_{n=0}^{\infty}\left\{\dot{u}_{\xi}^{(n)}, \dot{\bar{u}}_{\theta}^{(n)}, \dot{u}_{\xi}^{(n)}\right\} \cos n \theta+\left(\sigma_{0} a / E_{0}\right) \sum_{n=1}^{\infty}\left\{\dot{\bar{u}}_{\xi}^{(n)}, \dot{u}_{\theta}^{(n)}, \dot{\bar{u}}_{\xi}^{(n)}\right\} \sin n \theta$ $\left\{\dot{\varepsilon}_{\varepsilon m}, \dot{\varepsilon}_{\theta m}, \dot{\varepsilon}_{\varepsilon \theta m}, \dot{\varepsilon}_{\varepsilon s m}, \dot{\varepsilon}_{\theta s m}, \dot{\Phi}_{\xi}, \dot{\Phi}_{\theta}, \dot{\Phi}_{n}\right\}$ $=\left(\sigma_{0} / E_{0}\right) \sum_{n=0}^{\infty}\left\{\dot{e}_{\xi m}^{(n)}, \dot{e}_{\theta m}^{(n)}, \dot{\bar{e}}_{\xi o m}^{(n)}, \dot{e}_{\xi \xi m}^{(n)}, \dot{\bar{e}}_{\xi \xi m}^{(n)}, \dot{\varphi}_{\xi}^{(n)}, \dot{\bar{\varphi}}_{\theta}^{(n)}, \dot{\bar{\varphi}}_{n}^{(n)}\right\} \cos n \theta$ $+\left(\sigma_{0} / E_{0}\right) \sum_{n=1}^{\infty}\left\{\dot{\bar{e}}_{\xi m}^{(n)}, \dot{\bar{e}}_{\theta m}^{(n)}, \dot{e}_{\xi \theta m}^{(n)}, \dot{\bar{e}}_{\xi \xi m}^{(n)}, \dot{e}_{\xi \xi m}^{(n)}, \dot{\bar{\varphi}}_{\xi}^{(n)}, \dot{\varphi}_{\theta}^{(n)}, \dot{\varphi}_{n}^{(n)}\right\} \sin n \theta$

$\left\{\dot{\sigma}_{\xi}, \dot{\sigma}_{\xi}^{c}, \dot{\sigma}_{\xi}^{t}, \dot{\sigma}_{\theta}, \dot{\sigma}_{\theta}^{c}, \dot{\sigma}_{\theta}^{t}, \dot{\sigma}_{\xi \theta}, \dot{\sigma}_{\xi \theta}^{c}, \dot{\sigma}_{\xi \theta}^{t}, \dot{\sigma}_{\xi \xi}, \dot{\sigma}_{\xi \zeta}^{c}, \dot{\sigma}_{\theta 5}, \dot{\sigma}_{\theta \xi}^{c}\right\}$

$=\sigma_{0} \sum_{n=0}^{\infty}\left\{\dot{s}_{\xi}^{(n)}, \dot{s}_{\xi}^{c(n)}, \dot{s}_{\xi}^{t(n)}, \dot{s}_{\theta}^{(n)}, \dot{s}_{\theta}^{c(n)}, \dot{s}_{\theta}^{t(n)}, \dot{s}_{\xi \theta}^{(n)}, \dot{s}_{\xi \theta}^{c(n)}, \dot{\bar{s}}_{\xi \theta}^{t(n)}, \dot{s}_{\xi \xi}^{(n)}, \dot{s}_{\xi \xi}^{c(n)}, \dot{\bar{s}}_{\xi}^{(n)}, \dot{\bar{s}}_{\theta \xi}^{c(n)}\right\} \cos n \theta$

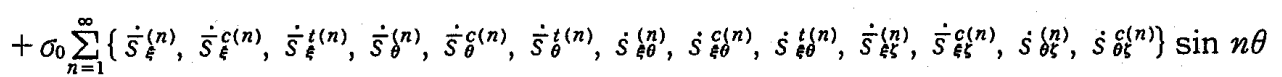

また, 分布荷重と温度変化（負荷）もフーリエ級数に展開する。

$\left.\begin{array}{l}\left\{\dot{P}_{\xi}, \dot{P}_{\theta}, \dot{P}_{\xi}\right\}=\left(\sigma_{0} h_{0} / a\right) \sum_{n=0}^{\infty}\left\{\dot{p}_{\xi}^{(n)}, \dot{\bar{p}}_{\theta}^{(n)}, \dot{p} \xi^{(n)}\right\} \cos n \theta+\left(\sigma_{0} h_{0} / a\right) \sum_{n=1}^{\infty}\left\{\dot{\bar{p}}_{\xi}^{(n)}, \dot{p}_{\theta}^{(n)}, \dot{\bar{p}}_{\xi}^{(n)}\right\} \sin n \theta \\ \dot{T}_{e}=\left(\sigma_{0} / E_{0} \alpha_{0}\right)\left(\sum_{n=0}^{\infty} \dot{t}_{e}^{(n)} \cos n \theta+\sum_{n=1}^{\infty} \dot{\bar{t}}_{e}^{(n)} \sin n \theta\right)\end{array}\right\}$

ここで, $\sigma_{0}, E_{0}, h_{0}, \alpha_{0}$ はそれぞれ代表応力, 代表縦弾 性係数, 代表殼厚, 代表線膨張係数である.

さて，これらのフーリエ展開式を前述の基礎式に代 入し，フーリエ係数に関する対応する式をつくり，こ れらの式から, 順次末知数を消去すると, 最後に平衡 方程式は変位 $\dot{u}_{\xi}, \dot{\bar{u}}_{\xi}, \dot{u}_{\theta}, \dot{\bar{u}}_{\theta}, \dot{u}_{\xi}, \dot{\bar{u}}_{\xi}$ および回転 $\dot{\varphi}_{\xi}$, $\dot{\bar{\varphi}}_{\xi}, \dot{\varphi}_{\theta}, \dot{\bar{\varphi}}_{\theta}$ に関する次の 10 元連立 2 階微分方程式に なる。なお，フーリェ級数の添字 $(n)$ は便宜上省略す る.

$$
A_{1} z^{\prime \prime}+A_{2} z^{\prime}+A_{3}=C
$$

ここに,

$$
z=\left\{\dot{u}_{\xi}, \dot{\bar{u}}_{\xi}, \dot{u}_{\theta}, \dot{\bar{u}}_{\theta}, \dot{u}_{5}, \dot{\bar{u}}_{\xi}, \dot{\varphi}_{\varepsilon}, \dot{\bar{\varphi}}_{\xi}, \dot{\varphi}_{\theta}, \dot{\bar{\varphi}}_{\theta}\right\}^{T}
$$

$A_{1} \sim A_{3}$ は $10 \times 10$ のマトリックスで殸の形状, 各層の 弾性係数, 各層の異方性主軸の方向から定まる係数で ある、Cはこれらのほかに, 分布荷重, 温度負荷およ びクリープによる見掛けの内力成分から定まる定数で ある。

温度負荷およびクリープによる見掛けの内力成分の 変化率の各フーリェ係数は, 式(21)，(27)から，それ ぞれ次のようになる。

$$
\begin{aligned}
& \sigma_{0} h_{0} \sum_{n=0}^{\infty}\left\{\dot{n}_{\xi}^{c(n)}, \dot{n}_{\theta}^{c(n)}, \dot{\bar{n}}_{\xi \theta}^{c(n)}, \dot{\bar{n}}_{\theta \xi}^{c(n)}, \dot{q}_{\xi}^{c(n)}, \dot{\bar{q}}_{\theta}^{c(n)}\right\}^{T} \\
& \quad \times \cos n \theta \\
& \quad+\sigma_{0} h_{0} \sum_{n=0}^{\infty}\left\{\dot{\bar{n}}_{\xi}^{c(n)}, \dot{\bar{n}}_{\theta}^{c(n)}, \dot{n}_{\xi \theta}^{c(n)}, \dot{n}_{\theta \xi}^{c(n)}, \dot{\bar{q}}_{\xi}^{c(n)}, \dot{q}_{\theta}^{c(n)}\right\}^{T}
\end{aligned}
$$

$$
\begin{aligned}
& \times \sin n \theta=\sum_{i=1}^{n} \int_{\zeta_{i-1}}^{\zeta_{t}}\left[L_{1}\right]\left[\bar{D}_{i}\right]\left[\dot{\bar{\varepsilon}}_{i}^{c}\right] d \zeta \\
& \left(\sigma_{0} h_{0}^{3} / a\right) \sum_{n=0}^{\infty}\left\{\dot{m}_{\varepsilon}^{c(n)}, \dot{m}_{\theta}^{c(n)}, \dot{\bar{m}}_{\xi \theta}^{c(n)}, \dot{\bar{m}}_{\theta \xi}^{c(n)}\right\}^{T} \cos n \theta \\
& +\left(\sigma_{0} h_{0}^{3} / a\right) \sum_{i=1}^{\infty}\left\{\dot{\bar{m}}_{\xi}^{c(n)}, \dot{\bar{m}}_{\theta}^{c(n)}, \dot{m}_{\xi \theta}^{c(n)}, \dot{m}_{\theta \xi}^{c(n)}\right\}^{r} \\
& \times \sin n \theta=\sum_{i=1}^{n} \int_{\zeta_{i-1}}^{\zeta_{t}}\left[L_{2}\right]\left[\bar{D}_{i}^{\prime}\right]\left\{\dot{\bar{\varepsilon}}_{i}^{i}\right]^{*} \zeta d \zeta \\
& \sigma_{0} h_{0} \sum_{n=0}^{\infty}\left\{\dot{n}_{\xi}^{t(n)}, \dot{n}_{\theta}^{t(n)}, \dot{\bar{n}}_{\varepsilon \theta^{t(n)}}, \dot{\bar{n}}_{\theta \xi}^{t(n)}, 0,0\right\}^{T} \cos n \theta \\
& +\sigma_{0} h_{0} \sum_{n=1}^{\infty}\left\{\dot{n}_{\xi}^{t(n)}, \dot{\bar{n}}_{\theta}^{t(n)}, \dot{n}_{\xi \theta}^{t(n)}, \dot{n}_{\theta \xi}^{t(n)}, 0,0\right\}^{T} \\
& \times \sin n \theta=\sum_{i=1}^{n} \int_{\zeta_{i-1}}^{\zeta_{i}}\left[L_{1}\right]\left[\bar{D}_{i}\right]\left\{\dot{\bar{\varepsilon}}_{i}^{t}\right\} d \zeta \\
& \left(\sigma_{0} h_{0}^{3} / a\right) \sum_{n=0}^{\infty}\left\{\dot{m}_{\xi}^{t(n)}, \dot{m}_{\theta}^{t(n)}, \dot{\bar{m}}_{\theta \varepsilon}^{t(n)}, \dot{\bar{m}}_{\theta \xi}^{t(n)}\right\}^{T} \cos n \theta \\
& +\left(\sigma_{0} h_{0}^{3} / a\right) \sum_{i=1}^{\infty}\left\{\dot{\bar{m}}_{\xi}^{t(n)}, \dot{\bar{m}}_{\theta}^{t(n)}, \dot{m}_{\xi \theta}^{t(n)}, \dot{m}_{\theta \xi}^{t(n)}\right\}^{T} \\
& \times \sin n \theta=\sum_{i=1}^{n} \int_{\xi_{i-1}}^{\xi_{i}}\left[L_{2}\right]\left[\bar{D}_{i}^{\prime}\right]\left\{\dot{\bar{\varepsilon}}_{i}^{t}\right\} * \zeta d \zeta
\end{aligned}
$$

式(31)の右辺の各層のクリープひずみ速度は, 式 （13）と式(17)によって各層の応力値から，また各層の 温度ひずみ速度は, 式(10)，(17)によって温度負荷か ら計算できる。また，積分にはシンプソンの $1 / 3$ 則を 用いる。

\section{3. 数 值計算法}

ある任意の計算段階に扔ける変位と回転の変化率は 式（29）を適当な境界条件のもとで解けば得られ，任意 
の時刻の解は，各段階の解の増分を積分することによ って得られる。しかしながら, 式(29)を厳密に理論解 析することは困難であるから，差分法によって数值的 に解を求める。この数值解法は著者らがすでに発表し た単層殼の場合 ${ }^{(9)}$ とほぼ同様であるので，ここでは紙 面の都合もあり省略する。

\section{4. 数 值 例}

数值例として，両端単純可動支持で，内側にステン レス鋼（SUS 316）を，外側にボイラ用炭素鋼（SB 42） を配した二層構造の閉じた円筒壳に，一様内圧が作用 する問題を取扱った（図 3 ）。

各層とも弾性変形に対しては等方性とし,クリープ 域に入って異方性が現れるものとした，また各層とも， 同一応力に対する直交異方性主軸方向のひずみ速度比 が $\dot{\varepsilon}_{1}^{c}: \dot{\varepsilon}_{2}^{c}: \dot{\varepsilon}_{3}^{c}=1: 4: 2$ の材料を仮定し, ステンレ ス鋼（第 1 層）とボイラ用炭素䥨（第 2 層）の主軸 1 方向と殸の $\xi$ 方向のなす角 $\varphi_{1}$ と $\varphi_{2}$ の 4 とおりの組 合せ $\left(\varphi_{1} / \varphi_{2}=0^{\circ} / 0^{\circ}, 45^{\circ} /-45^{\circ}, 0^{\circ} / 90^{\circ}, 90^{\circ} / 90^{\circ}\right)$ に対し て計算を行った. なおステンレス鋼の殸厚に対する比 は $h_{1} / h_{2}=0.3$ とした。

円筒殻の形状パラメー夕は次のようになる。

$$
\left.\begin{array}{l}
a=L, \xi=s / L, \rho=1 / 3, \rho^{\prime}=0, \gamma=0 \\
\omega_{\theta}=3, \omega_{\xi}=\omega_{\xi}^{\prime}=0
\end{array}\right\}
$$

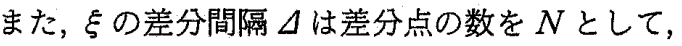

$$
\Delta=1 / 2(N-1)
$$

となる。

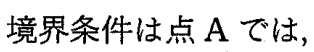

$$
\dot{U}_{\xi}=\dot{U}_{\theta}=\dot{U}_{\xi}^{\prime}=\dot{\Phi}_{\xi}=\dot{\Phi}_{\theta}=0
$$

ただし， $t=0$ のときは（`）を取り除くものとする。点 Bでは，単純可動支持の条件を用いて，

$$
\left.\begin{array}{l}
t=0: U_{\theta}=U_{\zeta}=\Phi_{\theta}=M_{\xi}=0, \\
N_{\xi}=(1 / 2) P^{-}(R-h / 2) \\
t>0: \dot{U}_{\theta}=\dot{U}_{\zeta}=\dot{\Phi}_{\theta}=\dot{M}_{\xi}=\dot{N}_{\xi}=0
\end{array}\right\}
$$

なお，式(27)の $\sigma_{0}$ は本計算では $\sigma_{0}=1$ とした。

SUS 316 とB 42 の材料定数は， $600^{\circ} \mathrm{C} に$ ける実 験より求めた次のものを用いた.

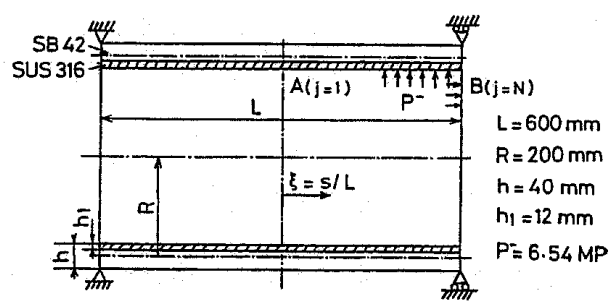

図 3 異方性 2 層円筒壳
SUS 316 :

$$
\begin{aligned}
& \left(E_{1}\right)_{1}=\left(E_{2}\right)_{1}=164 \mathrm{GPa},\left(\nu_{12}\right)_{1}=\left(\nu_{21}\right)_{1}=0.3 \\
& \left(G_{12}\right)_{1}=\left(G_{13}\right)_{1}=\left(G_{23}\right)_{1}=63.1 \mathrm{GPa} \\
& A_{1}^{\prime}=A_{1} e^{-\beta_{1} / T}=2.8 \times 10^{-19} \mathrm{MPa}^{-m_{1}}, m_{1}=6.69 \\
& C_{1}=0.51 \mathrm{~h}^{-1}, q=0.9 \mathrm{~h}^{-1} \\
& \text { B } 42: \\
& \left(E_{1}\right)_{2}=\left(E_{2}\right)_{2}=155 \mathrm{GPa},\left(\nu_{12}\right)_{2}=\left(\nu_{21}\right)_{2}=0.3 \\
& \left(G_{12}\right)_{2}=\left(G_{13}\right)_{2}=\left(G_{23}\right)_{2}=59.6 \mathrm{GPa} \\
& A_{2}^{\prime}=A_{2} e^{-\beta_{2} / T}=2.9 \times 10^{-13} \mathrm{MPa}^{-m_{2}}, m_{2}=5.53 \\
& C_{2}=0.5 \mathrm{~h}^{-1}, q_{2}=0.39 \mathrm{~h}^{-1}
\end{aligned}
$$

SB 42 :

異方性パラメー夕は， $\dot{\varepsilon}_{1}^{c}: \dot{\varepsilon}_{2}^{c}: \dot{\varepsilon}_{3}^{c}=1: 4: 2$ とした 場合, 次のようになる。

$$
\left.\begin{array}{l}
G_{1} / F_{1}=0.468, H_{1} / F_{1}=0.758 \\
L_{1} / F_{1}=M_{1} / F_{1}=N_{1} / F_{1}=2.23 \\
G_{2} / F_{2}=0.401, H_{2} / F_{2}=0.732 \\
L_{2} / F_{2}=M_{2} / F_{2}=N_{2} / F_{2}=2.13
\end{array}\right\}
$$

差分点数は, 子午線方向に $N=51$ とし, 板厚方向に は各層ごとに, 11 個の格子点を採用した。

クリープ変形中の各計算段階における時間増分 $\Delta t$ は，点 $\mathrm{A}$ における $M_{\xi}$ と $U_{5}$ の増分が，それぞれある 限界值 $M_{\xi}^{e} / 50, U_{5}^{e} / 20$ を超えないように制御した。こ こで, $M_{\xi}^{e}, U_{\xi}^{e}$ は点 A での $M_{\xi}$ と $U_{\xi}$ の初期の弾性解 である。

図 4 10 に計算結果 $\left(T=T_{0}=600^{\circ} \mathrm{C}\right)$ を例示する.

図 4 は, $\xi=0$ における変位 $U_{\zeta}$, 合モーメント $M_{\xi}$, $M_{\theta}$ の時間的変化を示している. 変位 $U_{\zeta}$ は, $\varphi_{1} / \varphi_{2}=$ $0^{\circ} / 0^{\circ}, 45^{\circ} /-45^{\circ}, 0^{\circ} / 90^{\circ}, 90^{\circ} / 90^{\circ}$ の順に小さくなって おり, $45^{\circ} /-45^{\circ}$ が $0^{\circ} / 0^{\circ}$ と $90^{\circ} / 90^{\circ}$ の中間にきてい る.また, $0^{\circ} / 90^{\circ}$ と $90^{\circ} / 90^{\circ}$ の差は小さい.

モーメント $M_{\xi}$ は，時間の経過とともに， $\varphi_{1} / \varphi_{2}=$ $0^{\circ} / 0^{\circ}, 45^{\circ} /-45^{\circ}$ の場合のほうが $\varphi_{1} / \varphi_{2}=90^{\circ} / 90^{\circ}, 0^{\circ} /$ $90^{\circ}$ の場合よりもやや小さくなっている(絶対值で).

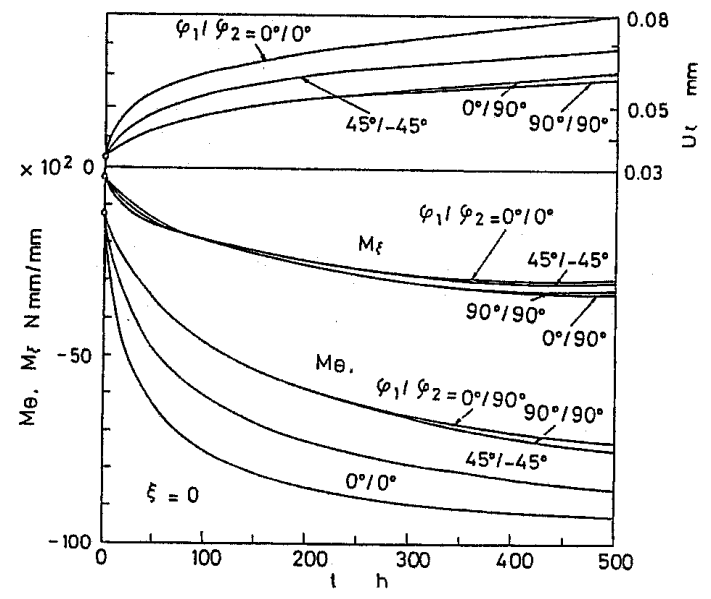

図 4 変位 $U_{\xi}$, 合モーメント $M_{\xi}, M_{\theta}(\xi=0)$ 
モーメント $M_{\theta}$ は， $M_{\xi}$ に比べて大きく，各 $\varphi_{1} / \varphi_{2}$ に よる差異は大きく, 大きさの順位は $M_{\xi}$ と逆になる。

図 5 は, 子午線断面の時間的変化を示す. $\varphi_{1} / \varphi_{2}=0^{\circ} /$ $0^{\circ}$ は $\varphi_{1} / \varphi_{2}=90^{\circ} / 90^{\circ}$ に比べ太短くなる. $45^{\circ} /-45^{\circ}$ の 変形はこれらのほぼ中間にくる， $\varphi_{1} / \varphi_{2}=0^{\circ} / 90^{\circ}$ と $90^{\circ} / 90^{\circ}$ は似た変形を示す.これは，クリープひずみが 出やすく, 厚さの支配的な軟鋼の層の異方性主軸の方 向が一致しているためである.

図 6 は, 合応力 $N_{\theta}$ の子午線方向分布の時間的変化 である、一般に，時間的変化は比較的小さいが， $\varphi_{1} / \varphi_{2}$ $=0^{\circ} / 0^{\circ}, 45^{\circ} /-45^{\circ}, 0^{\circ} / 90^{\circ}, 90^{\circ} / 90^{\circ}$ の順に大きくなっ ている、また, $0^{\circ} / 90^{\circ}$ と $90^{\circ} / 90^{\circ}$ の場合はほほ等しい.

図 7 は, 世ん断力 $Q_{\xi}$ と $Q_{\theta}$ の子午線方向分布の時

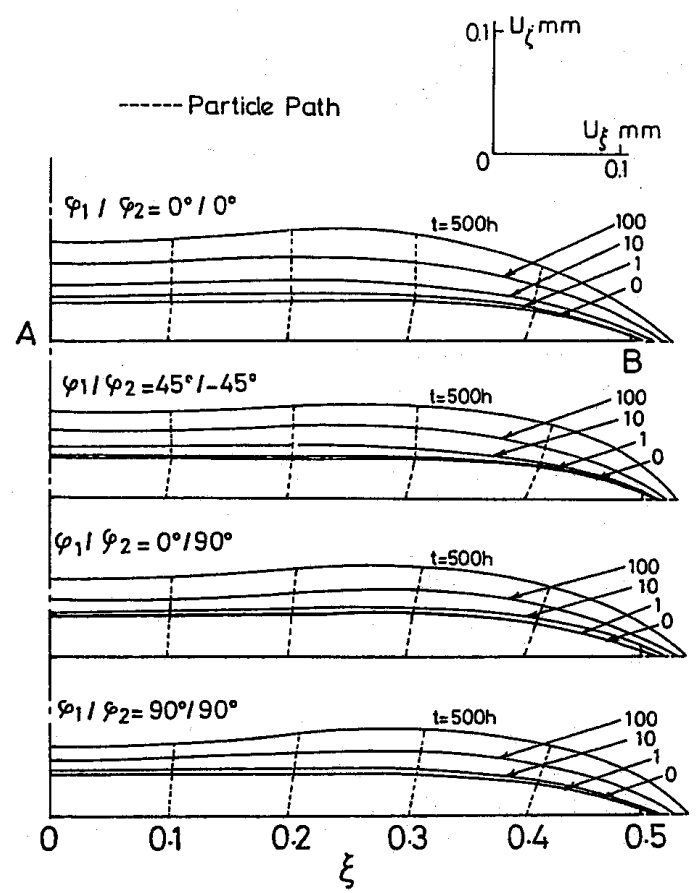

図 5 子午線断面の変形

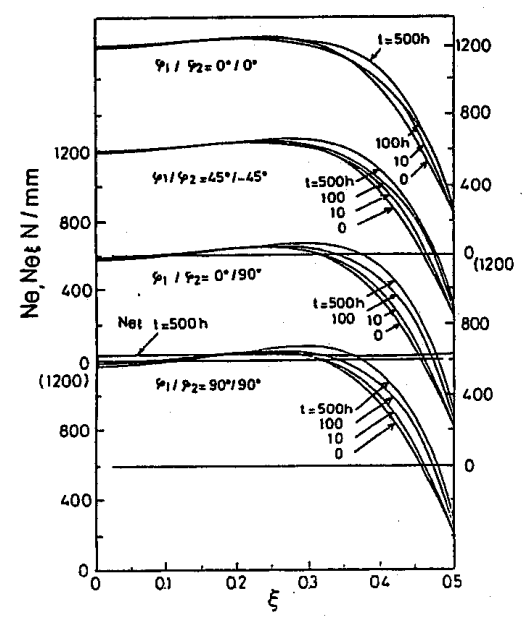

図 6 合応力 $N_{\theta}, N_{\theta \xi}$ の子午線方向分布

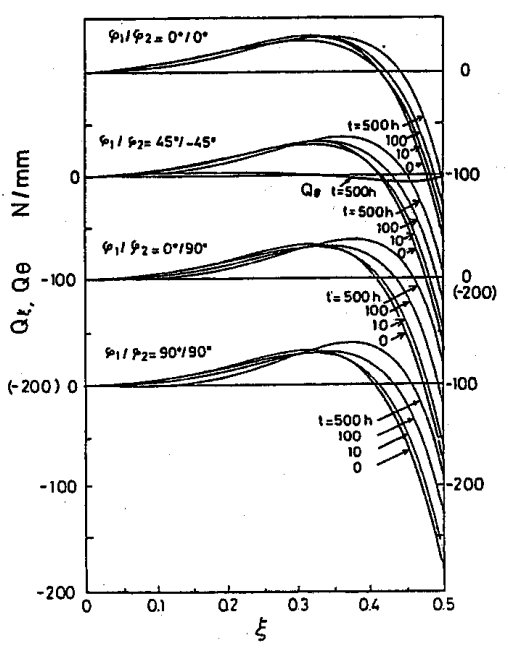

図 7 合応力 $Q_{\xi}, Q_{\theta}$ の子午線方向分布
間的変化である。 $\varphi_{1} / \varphi_{2}$ による $Q_{\xi}$ の変化の傾向は $N_{\theta}$ と類似している.

図 8 抢よび図 9 は，それぞれ合モーメント $M_{\xi}$ 抢よ び $M_{\theta}$ の子午線方向分布の時間的変化である.いずれ も時間的変化は大きい. $\varphi_{1} / \varphi_{2}$ による時間的変化は, $M_{\xi}$ は $0^{\circ} / 0^{\circ}, 45^{\circ} /-45^{\circ}, 90^{\circ} / 90^{\circ}, 0^{\circ} / 90^{\circ}$ の順に大きく, また $M_{\theta}$ はこの順に小さくなっている.なお $M_{\xi} の 0^{\circ} /$ $90^{\circ}$ と $90^{\circ} / 90^{\circ}$ との間にはあまり差がない.

なお, 図 5〜9において, $\varphi_{1} / \varphi_{2}=45^{\circ} /-45^{\circ}$ の場合に は，異方性のために，時間の経過とともに， $U_{\theta}, N_{\varepsilon \theta}$, $N_{\theta \xi}, Q_{\theta}, M_{\xi \theta}, M_{\theta \xi}$ が現れるが，それらの值は極めて小 さい.

図 10 は応力 $\sigma_{\xi}, \sigma_{\theta}$ の $\xi=0$ に扮ける款厚方向分布 の時間的変化である。応力は, 時間とともに, SB 42 側 では緩和しているのに対し, SUS 316 側では増加し, SB 42 と SUS 316 の境界面における不連続は大きく なる。 $\varphi_{1} / \varphi_{2}$ に対する時間的変化は, $\sigma_{\xi}$ は $0^{\circ} / 0^{\circ}, 45^{\circ} /$ $-45^{\circ}, 90^{\circ} / 90^{\circ}, 0^{\circ} / 90^{\circ}$ の順に大きくなっているが, $\sigma_{\theta}$ はこの順に小さくなっている。また， $0^{\circ} / 90^{\circ}$ と $90^{\circ} / 90^{\circ}$ の変化量にはほとんど差がない.

本計算には，熊本大学情報処理センターの FACOM M-360を使用し，それぞれの数值例の計算時間は約 $15 \min$ である。

\section{5. 結言}

本論文では，各層に直交異方性があり，異方性主軸 の方向の異なる層を重ね合わせた多層回転対称壳のク リープ変形問題を取扱い, 応力と変形の時間的変化の 様子を解析する方法を示した，各層のクリープの構成

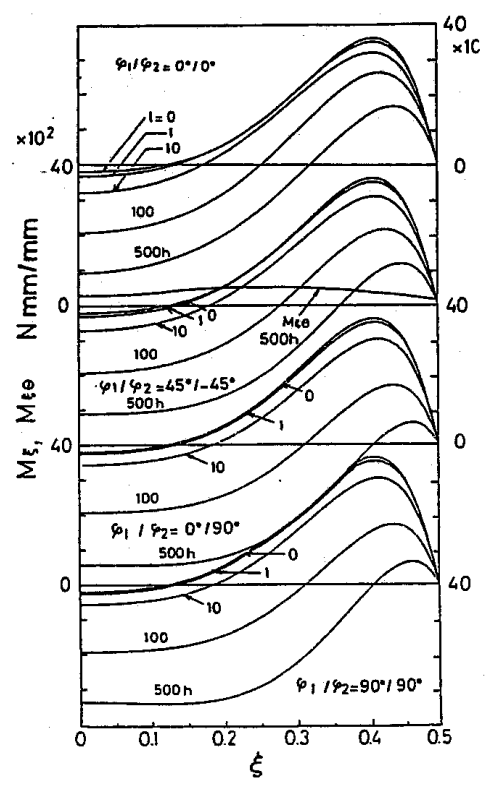

図 8 合モーメント $M_{\xi}, M_{\xi \theta}$ の 子午線方向分布 


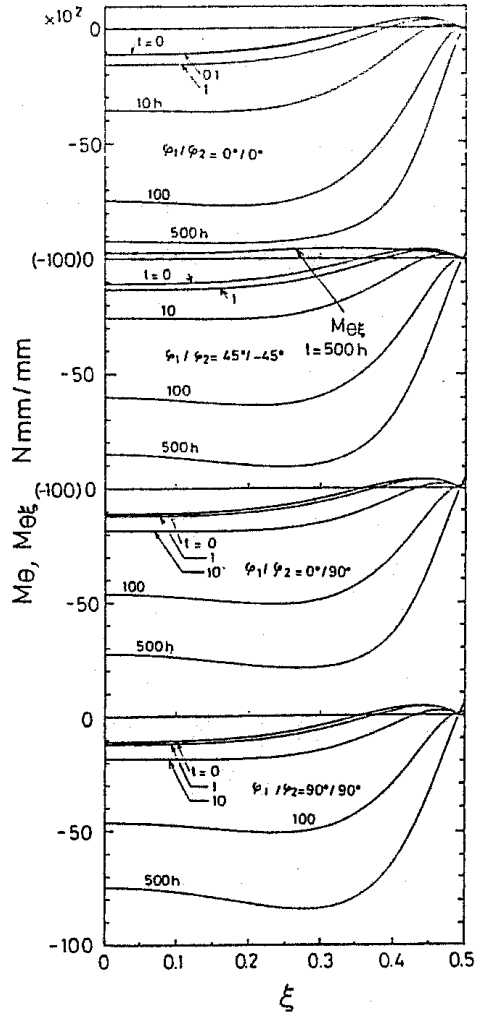

図 9 合モーメント $M_{\theta}, M_{\theta \xi}$ の子午線 方向分布

関係には, 温度効果をアレニウスの式で表した McVettyの式を, Hill の直交異方性塑性理論になら って，多軸応力状態に拡張したものを用いた。 また，厚 肉の場合に考慮すべきせん断変形には, ReissnerNaghdi の理論を用いた。数値解法には差分法を適用 した.

数值例として, 直交異方性を有するSB 42 と SUS 316 から成る二層構造の円筒殻に, 一様内圧が作 用する場合を $\varphi_{1} / \varphi_{2}$ の值を変えて解析した. その結果, 変形と内力は, $\varphi_{1} / \varphi_{2}$ の值によって著しく異なること が認められた。

\section{文献}

(1) Penny, P. K., J. Mech. Eng. Sci., 6-1 (1964), 44.
（2）村上・鉿木，機論，38-316（昭 47)，3048。

（3）竹園・ほか 2 名，機論, 38-310 (昭 47), 1183.

（4）竹園・ほ加 2名, 機論, 39-327 (昭 48), 3304.

（5）竹園・ほか 2 名，機論，41-341 (昭 50), 51.

（6）矢川・ほか 3 名，機論，41-350 (昭 50)，2784.

(7) Takezono, S. and Uchida, K., Proc. Int. Conf. Eng. Aspect Creep, 2 (1980), 57.

(8) Takezono, S. and Fujioka, S., Creep in Structures, (1981), 128, Springer-Verlag.

（9）竹園・内田, 機論, 49-441, A (昭 58)，618.

(10) Takezono, S. and Migita, K., Proc. Int. Conf. Creep, (1986), 429.

（11）竹園 - 右田, 機論, 53-485, A (昭 62), 97.

(12) Penny, P.K. and Marriott, D. L., Design for crrep, (1971), 7, McGraw.Hill.

(13) Reissner, E., Am. J. Math., 63-1 (1941), 177.

(14) Naghdi, P. M., Q. Appl. Math., 14-4 (1957), 396. 


\section{討 論}

〔質問〕村上澄 男〔名古屋大学工学部〕

殼の工学的クリープ変形問題に見られるほとんどす べての要因を考慮し，その有効な数值解析法を開発さ れたことに敬意を表す。以下の点につき，ご教示願い たい.

（1）殼の層間の接続条件は，境界面をはさんでの 変位の連続条件と考えてよろしいか。

（2） 2 層殼の非弾性変形問題では, 合応力, 合モ ーメント, 殼中央面の変位などのような平均的物理量 は, 各層単独の挙動からほほ添推定できると思われる。 これに対して, 疲労寿命の推定等のためには局所的応 力分布が重要である。層間せん断応力は, 図 $100 \sigma_{\xi}$, $\sigma_{\theta}$ と比べてどの程度の大きさか。

（3）図10から判断して, 一定荷重を受ける 2 層 殼がクリープのような非線形性の強い変形を示す場
合, 弱いほうの構造は有効に寄与しないと解釈してよ ろしいか。

（4）この種の問題の数值解析に対して，この論文 の方法と有限要素法等との優劣を比較するとどのよう になるか。

〔回答〕（1）境界面でひずみが連続であると考 えている.

（2）層間せん断応力は図 10 の $\sigma_{\xi}, \sigma_{\theta}$ の 1 2 割程 度である。

（3）殼厚全体の強度に対する各層の寄与は, 一般 に, 各層の強度のほかに厚さと配置によっても異なる ものと考えられる.

（4）同じ例題を有限要素法で解析していないの で, 計算機の必要容量, 計算時間, 計算精度等の優劣 を比較することは困難である. 